\title{
A pragmatic method for costing implementation strategies using time- driven activity-based costing
}

\author{
Zuleyha Cidav ${ }^{1,2^{*}}$ (D), David Mandell ${ }^{1,2}$, Jeffrey Pyne ${ }^{3,4,5,6}$, Rinad Beidas ${ }^{1,2,7,12,13}$, Geoffrey Curran ${ }^{8,9,10}$ and \\ Steven Marcus ${ }^{1,2,11}$
}

\begin{abstract}
Background: Implementation strategies increase the adoption of evidence-based practices, but they require resources. Although information about implementation costs is critical for decision-makers with budget constraints, cost information is not typically reported in the literature. This is at least partly due to a need for clearly defined, standardized costing methods that can be integrated into implementation effectiveness evaluation efforts.

Methods: We present a pragmatic approach to systematically estimating detailed, specific resource use and costs of implementation strategies that combine time-driven activity-based costing (TDABC), a business accounting method based on process mapping and known for its practicality, with a leading implementation science framework developed by Proctor and colleagues, which guides specification and reporting of implementation strategies. We illustrate the application of this method using a case study with synthetic data.

Results: This step-by-step method produces a clear map of the implementation process by specifying the names, actions, actors, and temporality of each implementation strategy; determining the frequency and duration of each action associated with individual strategies; and assigning a dollar value to the resources that each action consumes. The method provides transparent and granular cost estimation, allowing a cost comparison of different implementation strategies. The resulting data allow researchers and stakeholders to understand how specific components of an implementation strategy influence its overall cost.

Conclusion: TDABC can serve as a pragmatic method for estimating resource use and costs associated with distinct implementation strategies and their individual components. Our use of the Proctor framework for the process mapping stage of the TDABC provides a way to incorporate cost estimation into implementation evaluation and may reduce the burden associated with economic evaluations in implementation science.
\end{abstract}

Keywords: Costing, Economic evaluation, Implementation strategies, Time-driven activity-based costing

\footnotetext{
* Correspondence: zcidav@upenn.edu

'Department of Psychiatry, Perelman School of Medicine, University of Pennsylvania, Philadelphia, PA, USA

${ }^{2}$ Leonard Davis Institute of Health Economics, University of Pennsylvania, Philadelphia, PA, USA

Full list of author information is available at the end of the article
}

(c) The Author(s). 2020 Open Access This article is licensed under a Creative Commons Attribution 4.0 International License, which permits use, sharing, adaptation, distribution and reproduction in any medium or format, as long as you give appropriate credit to the original author(s) and the source, provide a link to the Creative Commons licence, and indicate if changes were made. The images or other third party material in this article are included in the article's Creative Commons licence, unless indicated otherwise in a credit line to the material. If material is not included in the article's Creative Commons licence and your intended use is not permitted by statutory regulation or exceeds the permitted use, you will need to obtain permission directly from the copyright holder. To view a copy of this licence, visit http://creativecommons.org/licenses/by/4.0/. The Creative Commons Public Domain Dedication waiver (http://creativecommons.org/publicdomain/zero/1.0/) applies to the data made available in this article, unless otherwise stated in a credit line to the data. 


\section{Contributions to the literature}

- Implementation strategies help healthcare settings to adopt evidence-based practices, but stakeholders often cite their cost as a barrier. More detailed information about costs, including information that aids logistical decisions, could boost adoption

- We apply an established method from the business accounting literature, time-driven activity-based costing, to implementation by combining it with the Proctor et al. framework, an established framework often used in implementation effectiveness evaluations to guide the specification and reporting of implementation strategies.

- This method can serve as a pragmatic tool to operationalize the conduct of implementation activities, determine resource consumption, and estimate associated costs. It could facilitate routine economic evaluations in implementation research.

\section{Background}

The ability to assess the cost-effectiveness of evidence-based practices (EBPs) is critical to ongoing efforts to improve healthcare quality and reduce healthcare costs. This includes assessing the costs associated with implementation strategies or the activities required to achieve successful uptake of EBPs. Cost concerns are associated with reduced stakeholder willingness to implement EBPs, and thus, they have emerged as the most significant and least modifiable barrier to EBP implementation [1]. In particular, despite growing evidence that EBPs can be cost-effective over time, start-up costs are frequently cited as a major barrier to implementation [2, 3]. These costs may include external trainer and consultant time, internal staff time spent on training and receiving consultation, data collection and reporting, additional supervision, fidelity monitoring, and spending on travel, books, and other required materials.

In keeping with the fact that a clear understanding of implementation costs is important for determining the feasibility and achieving buy-in from stakeholders, many implementation frameworks highlight cost as an important factor to consider when evaluating implementation approaches and outcomes $[3,4]$. However, there is little guidance with regard to how to pragmatically estimate these costs [5-7]. Few implementation science studies have considered costs, and even fewer have compared the costs of various implementation strategies, despite numerous calls for this type of research $[8,9]$. For example, a systematic review of 235 implementation studies found that only $10 \%$ provided information about implementation costs [9]. In the rare cases where implementation costs are reported, usually they are presented as broad categories of spending (e.g., personnel, supplies, travel) $[10,11]$. This approach uses total expenditure data (or program budget) to provide gross average estimates of costs. This information is helpful, but it does not offer sufficient detail to guide important decisions.

There have been several efforts to break down aggregate costs into more specific estimates of the resources consumed by various implementation activities. Recently, Ritchie et al. estimated the cost of facilitation as a strategy to implement integrated primary care and mental healthcare services [12]. They listed various activities performed by facilitators and stakeholders and regularly collected data on time spent on each activity to estimate costs. Similarly, the Cost of Implementing New Strategies (COINS) framework was designed to map costs onto pre-determined implementation stages and activity categories associated with the Stages of Implementation Completion (SIC) measure, an 8-stage tool to assess implementation process and milestones [13]. Liu et al. have broken down the implementation strategy of provider education into activities such as preparing and producing educational materials, providing introductory educational conferences, in-person or video conferencing for supervision and consultation, ongoing seminars, and academic detailing [14]. O'Beirne et al. broke down recruiting clinics into activities such as revising mail-out responses and preparing for and attending meetings [10].

We sought to build on these efforts by introducing a process-based approach for costing implementation strategies, borrowed from business settings, and mapping it on to an established implementation science framework that can be used to guide effectiveness evaluations. Time-driven activity-based costing (TDABC) is a micro-costing method that is widely used in business settings to determine various ways in which the structure of business activities can be redesigned to realize potential areas for improvement [15]. It does this by delivering more detailed, accurate, and transparent activity cost information that is more relevant and actionable for decision-making $[10,11,16-18]$. Its utility has led to its adoption by other sectors, including the service and healthcare industries $[15,19-22]$. TDABC is well matched to implementation science's focus on processes and procedures in the uptake of EBPs. The Proctor framework for identification, specification, and 
reporting of implementation strategies offers a guideline to be followed when evaluating the effectiveness of implementation strategies [23]. Our proposed method integrates TDABC and the Proctor framework and provides a step-by-step approach to costing implementation strategies. In this paper, we outline the method in detail and present a case study of its application.

\section{Method and case study}

\section{Time-driven activity-based costing}

TDABC is a process-based micro-costing methodology that provides detailed cost data through the use of process maps. It is particularly useful when costs are driven mainly by personnel time, hence the name "time-driven" [20]. Under the TDABC framework, a process is a chain of activities performed in combinations to achieve a certain purpose, such as delivering a service. Processes can be defined at any level of detail, starting at the most abstract and becoming more concrete by successive decomposition [24]. Specifically, each process can be broken down into a set of related procedures that describe the exact steps to complete the process.

The general steps of TDABC, adapted from Kaplan et al. [20], are (1) creation of a process map that outlines the procedures that comprise the process, where the procedures are defined as quantifiable events; (2) determination of the frequencies of those defined procedures; (3) estimation of the time-per-unit for the procedure, which specifies how long it takes to execute one unit of the procedure (one single event); (4) calculation of the total time spent to complete the procedure (i.e., multiplying the frequency of the procedure's occurrence by the time-per-unit); (5) estimation of the cost per hour for each resource used; and (6) calculating the total cost of the procedure by multiplying the total procedure time (in hours) by the per-hour cost of resources. Summing the costs of procedures that comprise a process yields the process costs. In short, TDABC requires information on who (personnel completing the task) does what (specific activities performed), when (timing), and how often (the frequency, intensity and/or duration of the activity) [25].

TDABC is a modified version of activity-based costing $(\mathrm{ABC})$, an earlier approach to cost accounting that relies on employee self-reported data to determine the percentage of time spent on the activities, or on ongoing activity time logs $[20,21]$. Data generated in that manner can be timeconsuming and costly to collect as well as difficult to validate $[15,19,20]$. TDABC changes how the time data are collected and modifies the calculation of activity costs $[15,20,26,27]$. To do so, it requires only three parameters: (1) frequency of the activity (i.e., how many times the activity occurs), (2) time required to perform one single event of the activity (e.g., 1 hour), and (3) per-hour price of the resources (e.g., personnel time) used to perform the activity [16, 17]. This approach reduces the burden of time use data collection [20-22, 26] and the risk of inaccurate or subjective employee-reported data because the frequency and average duration of activities can typically be observed objectively (e.g., by checking the time stamp on a recorded consultation session) costs $[15,19-21,26,27]$, or they can be standardized and specified in advance in a way that does not require observation (e.g., a standard 3-h training session). TDABC has been shown to be practical when applied in various organizational settings including healthcare $[15,19,22,28]$. Under TDABC, rough accuracy (i.e., how close your cost estimate is to the actual cost) is sufficient; precision (i.e., the number of decimal places included in the estimation) is not critical [20, 21]. In addition, because TDABC relies on granular, procedure-level costs as opposed to broad categories, it allows stakeholders to see what resources were used for what purposes. For example, it disaggregates the costs of an output (e.g., a consultation meeting) into the specific inputs or items consumed during its implementation (e.g., clinician time, travel costs, consumable supplies), which can help identify areas for cost savings.

\section{Proctor et al. framework for implementation strategy specification and reporting}

Implementation strategies in existing literature have been criticized as being poorly described, inconsistently labeled, and insufficiently detailed [8, 23]. In response, many guidelines emerged that sought to develop a more standardized language for implementation strategies and other aspects of implementation $[23,29-31,23,29-32]$. One set of guidelines, proposed by Proctor and colleagues [23], suggests that when studying implementation strategies, researchers should first name or label the implementation strategies in ways that are consistent with the published literature; then define them conceptually (i.e., give a general sense of what the strategy might involve); and finally, operationalize them by carefully specifying the following elements: (1) actor (i.e., who enacts the strategy?), (2) action(s) (i.e., what are the specific actions, steps, or processes that need to be enacted?), (3) action target (i.e., what constructs are targeted?), (4) temporality (i.e., when is the strategy used?), (5) dose (i.e., 
what is the intensity?), (6) implementation outcome (i.e., what implementation outcome(s) are likely to be affected by each strategy?), and (7) justification (i.e., what is the empirical, theoretical, or pragmatic justification for the choice of an implementation strategy?) [23, 33]. This approach has increasingly been applied in implementation research [6,33,34], and the Standards for Reporting Implementation Studies (STARI), a widely accepted checklist for transparent and accurate reporting of studies that evaluate implementation strategies [32], recommends this framework to describe implementation strategies.

\section{Costing implementation strategies using the TDABC and Proctor et al. framework}

The information on implementation strategies generated by following the Proctor et al. framework aligns directly with the information needed for a TDABC approach to costing. This enables a novel application of the Proctor framework in assessing implementation strategy costs. In short, both approaches require specification of who, does what, when, how often, and for how long [23]. Following the Proctor et al. framework and generating information on actor (who), action (does what), temporality (when), and dosage (how often, for how long) allows it to guide application of the first step in the TDABC approach: process mapping to operationalize each implementation strategy to be used in a given project.

In our proposed method, we conceptualize an implementation strategy as a process associated with executing a series of specific procedures (actions) performed by utilizing human resources (actors) and non-human resources. Because the cost of an implementation strategy is primarily driven by the time spent by the actors executing its specific actions, for simplicity, we describe how our proposed approach would be used to estimate the cost of personnel resources, which is usually the largest time-driven cost item in implementation efforts. However, the estimation of the time-driven cost of nonpersonnel resources (e.g., office space rented monthly to perform a specific implementation strategy action) can be carried out similarly. If those costs are to be included, any non-personnel resources with time-driven costs would be listed for each action, alongside the actors.

Next, we use a case study and synthetic data to demonstrate how this step-by-step method can be used in implementation science.

\section{Results}

Case study: costing "General Practice Facilitation," a multicomponent evidence-based implementation strategy General practice facilitation is an evidence-based implementation strategy for assisting healthcare practices in implementing EBPs or improving in areas such as patient access and care [35]. Usually outside experts, practice facilitators, or practice coaches work closely with healthcare practices to develop the capacity for sustained change and improvement. In this case study, a primary care practice receives practice facilitation using multiple implementation strategies for the implementation of two psychotherapy EBPs. Using the adapted TDABC framework for costing implementation strategies and the Proctor et al. framework as a guide, we implement our method as outlined below. Appendix 1 presents a sample TDABC costing matrix that serves as a blueprint for this process, and Table 1 displays a completed matrix for our case study.

\section{Step 1: Name the implementation strategy and list the associated actions, actors, and temporality}

In this first step of process mapping, the Proctor et al. framework is used to specify the procedures that comprise the implementation strategy. This includes the following:

1) Naming the implementation strategy in a way that is consistent with the existing guidelines and taxonomies in the literature [30,36,37].

2) Identifying and listing the strategy actions (i.e., what needs to be done) required for the successful execution of the implementation strategy.

3) Identifying the temporality of each action (when the action is performed; start and end dates of the actions).

4) Identifying and listing the actors (who will enact the actions) involved in each action.

The initial goal is to fully understand and document the implementation process and create a blueprint of the implementation strategy. This requires careful study of the implementation protocol and detailed operational information from the key personnel (e.g., project managers, facilitators) who are most familiar with the implementation project, to ensure comprehensiveness and accuracy. Key personnel directs the process-mapping component. They start by identifying the high-level implementation strategies and then drill down into the actions that occur in each strategy. An action is a discrete activity involving one or more resourcespersonnel and/or equipment. Developing a process map enables key personnel to describe all the main actions involved in delivering the implementation strategy along with the resources consumed at that action.

As shown in Table 1, we specified five discrete implementation strategies (column I): initial work for site 


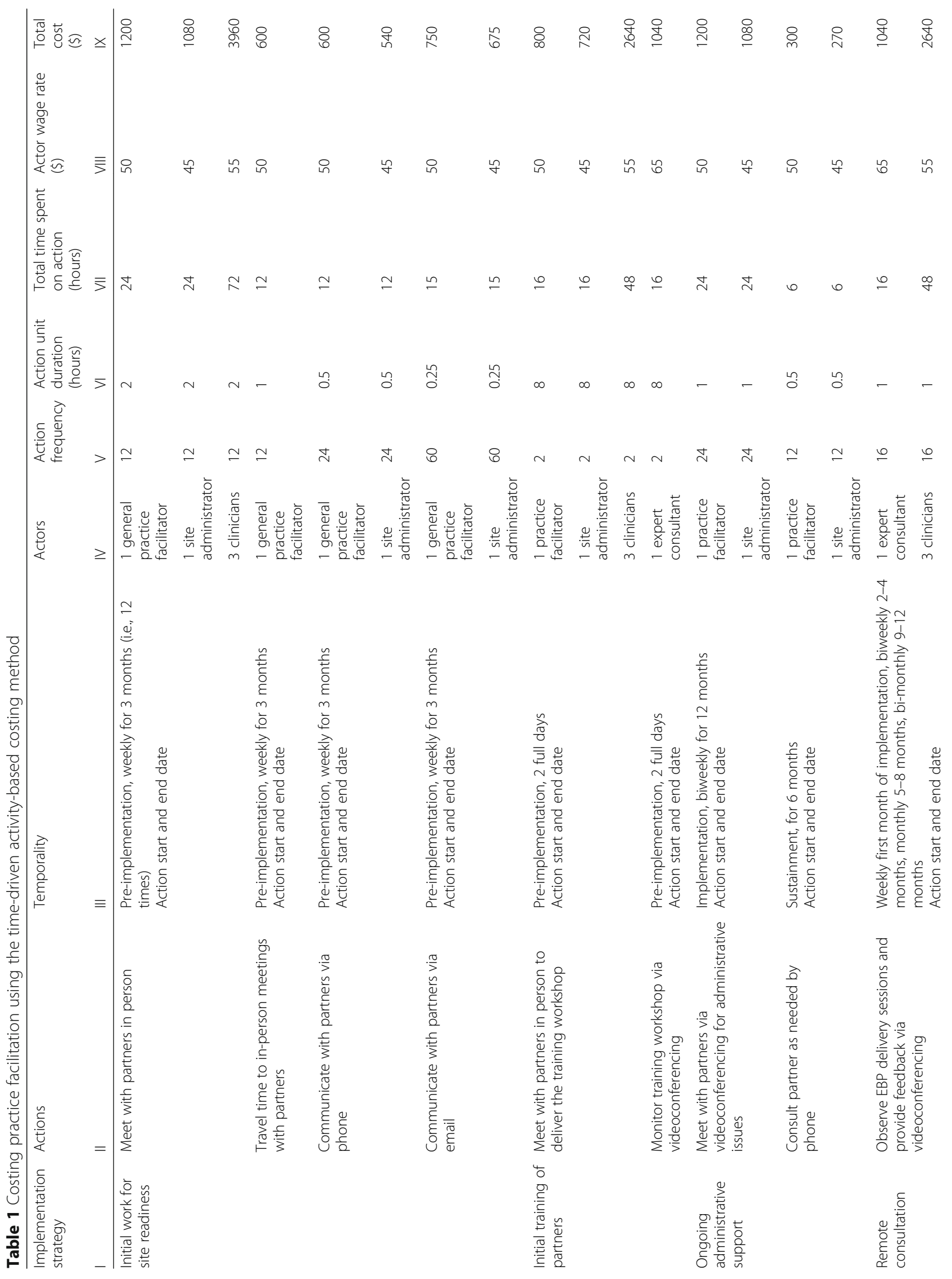




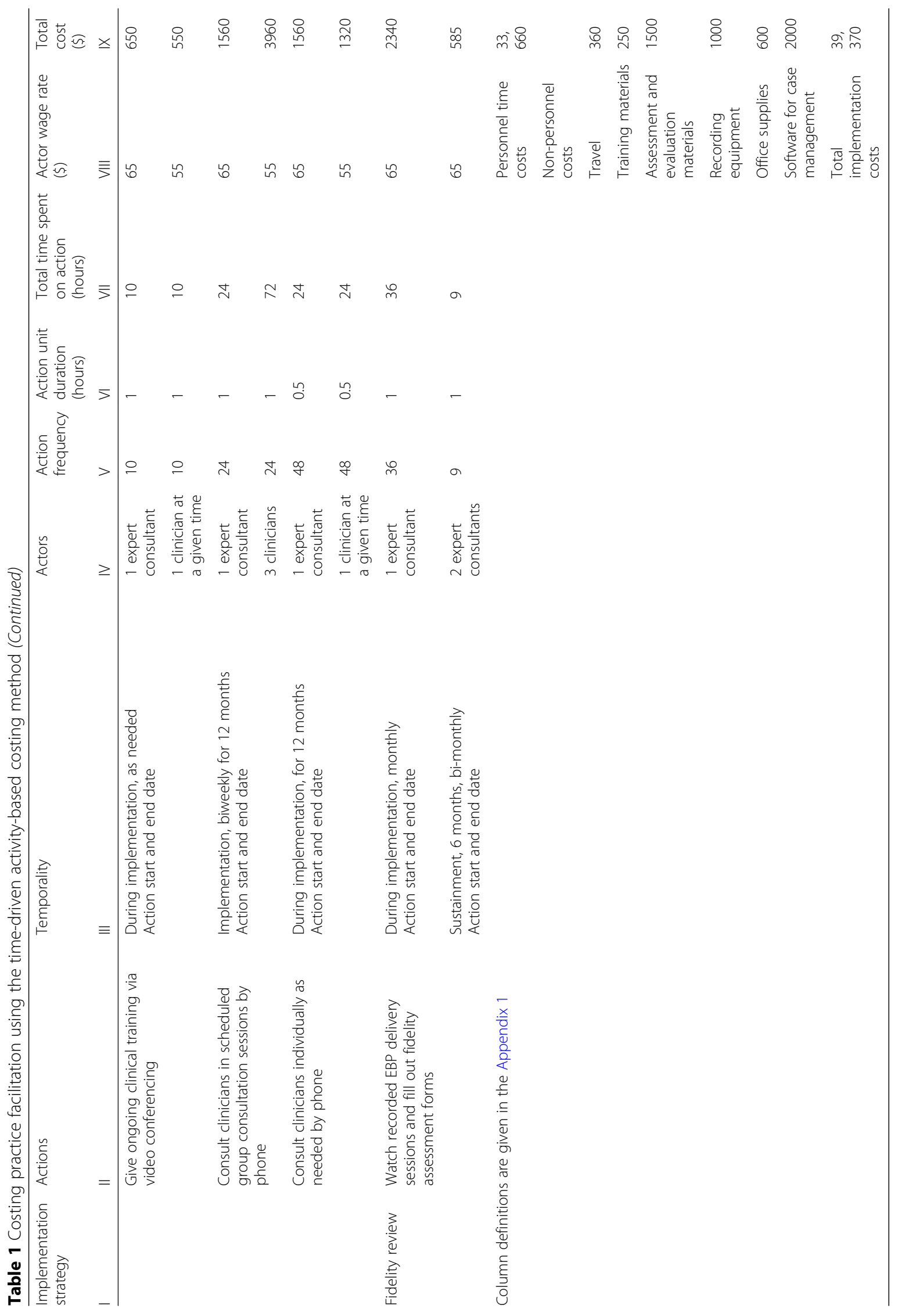


readiness, initial training of the partners, ongoing administrative support, remote consultation, and fidelity review. A complete list of main actions is shown in column II, including activities such as holding in-person and videoconference meetings, making phone calls and exchanging emails, conducting an initial training workshop, conducting consultation and feedback sessions with clinicians via videoconferencing, and filling out fidelity assessment forms.

Column III describes temporality, or when each implementation strategy and its associated actions occur. Implementation efforts span a total of 24 months, including pre-implementation, implementation, and sustainment periods. For example, initial work for site readiness takes place during the first 3 months (pre-implementation), as does the training of the partners in a one-time workshop that lasts 2 days. Ongoing technical and administrative support, remote consultation, and fidelity review take place during the 12-month implementation period and the 6-month sustainment period. As noted in Table 1, temporality should include information on the specific start and end dates of the activities; this defines the observation period for which the frequency of the actions will be determined. The implementation actors (or personnel resources) are listed in column IV. In our case study, they include a team of one general practice facilitator, one expert liaison psychologist, one site administrator, and three site clinicians.

Implementation researchers can prepare a TDABC template prospectively by identifying implementation strategies, actions, temporality, actors, planned frequency, and unit duration of actions. Because implementation strategies are often standardized (e.g., procedure frequency and duration are decided beforehand) [38], such a TDABC template can be filled out a priori based on the study protocol and serve as a blueprint prior to the start of implementation.

When implementation efforts start, the TDABC template serves as a monitoring tool to track the real-time implementation process and can be used to track the strategy as delivered. The initial estimation of costs in the matrix can then be compared to the costs of the implementation strategies as actually delivered $[8,39-41]$ to compare estimated versus actual costs. Direct observation of the actions might reveal variations in the strategy as planned, allowing for documentation of required revisions in the initial template and yielding a map of the strategy as delivered in real time. This element of the method is consistent with calls within implementation science to determine how strategy delivery varies from what was originally intended and to assess the implications of this variation for implementation outcomes. The TDABC process map can be used as a monitoring tool for the delivery of implementation strategies.

Step 2: Determine the frequency and average duration of each implementation action by actors, as well as actors' total time spent on each action

Since information on activity frequency (i.e., how many times the activity occurs) and average duration (i.e., how long it takes to perform one unit of activity) is central to TDABC, process map actions need to be operationalized as concrete, observable, single events. For every action, a "unit of action" should be specified so that action frequency and average duration can be quantified. For example, a unit action can be delivering one training session. The average "unit duration" is then how long it takes to complete the unit action on average (e.g., the average duration of one training session). The total time spent on an action is determined by multiplying the observed action frequency within the timeline that the action takes place (temporality) and the action unit duration (the average time spent to perform one unit of action).

In Table 1, for each implementation strategy action, columns V and VI show its frequency and unit duration, respectively. Additional detail regarding how the action frequencies and unit durations are specified may be found in Appendix 2. For example, for the action "Provide feedback via videoconferencing," the unit action is one single videoconference call. The frequency of action specifies how many videoconference calls take place, and the action unit duration is the average length of a single videoconference call. The values in column VII are derived by multiplying the action frequency by each actor (columns IV and V) and unit duration (column VI), yielding total time spent on the action by the specific actor.

\section{Step 3: Determine the price per hour of each actor}

Information needed for calculating the price per hour of the resources comes from the project budget. Wage rate, or price per unit hour, is the total spending on the resource (e.g., salary) divided by the total hours provided by the resource.

In Table 1, column VII presents the price per actor (personnel) hour, which is calculated by dividing each person's annual salary (including employee benefits) by 2080 annual work hours. In the case of consultant time, this would represent the hourly billing rate. 
Step 4: Determine non-personnel, fixed resources, and their associated expenses

Non-personnel resources whose costs are fixed and not time-driven (such as consumable equipment, supplies, and technology required to perform the actions) are itemized and the expense of each item is determined. This information usually can be obtained from the project budget.

In Table 1, non-personnel resources shared across actions include spending on items like training materials, assessment and evaluation materials, recording equipment, office supplies, and software for case management. These are fixed costs rather than timedriven, so we report their costs separately at the bottom of Table 1.

\section{Step 5: Calculate total costs}

The total time spent on each action by each actor (step 2) is multiplied by the price per hour of the actor (step 3) in order to obtain the action cost for each actor. The sum of action costs yields the cost of personnel resources for the implementation strategy. Non-personnel, fixed costs are added to derive the total cost of the implementation strategy.

Finally, column IX in Table 1 shows the action costs. These are calculated by multiplying the total time spent on the action (column VII) by the price per hour (column VIII). Total personnel time costs, non-personnel expenses, and total cost of the implementation project are then summed and listed at the bottom of the table.

\section{Transforming the TDABC matrix into user-friendly reports and decision tools}

The detailed information captured in the final TDABC resources matrix (Table 1) can be transformed into numerous visual representations of project costs that can help stakeholders to understand both personnel and non-personnel resource allocation and associated costs. Figures 1, 2, 3, and 4 illustrate sample infographics that can be useful in assessing costs and making decisions. Figure 1 focuses on the composition of total costs (cost by personnel, the cost for each non-personnel item). Figure 2 illustrates the costs for each of the five implementation strategies used in our case study, including what portion of the costs of each strategy is devoted to each actor or personnel member. Figure 3 shows the cost of each specific action that is performed as part of each implementation strategy, and Fig. 4 shows costs by implementation phase, including the cost of specific personnel time within each phase.

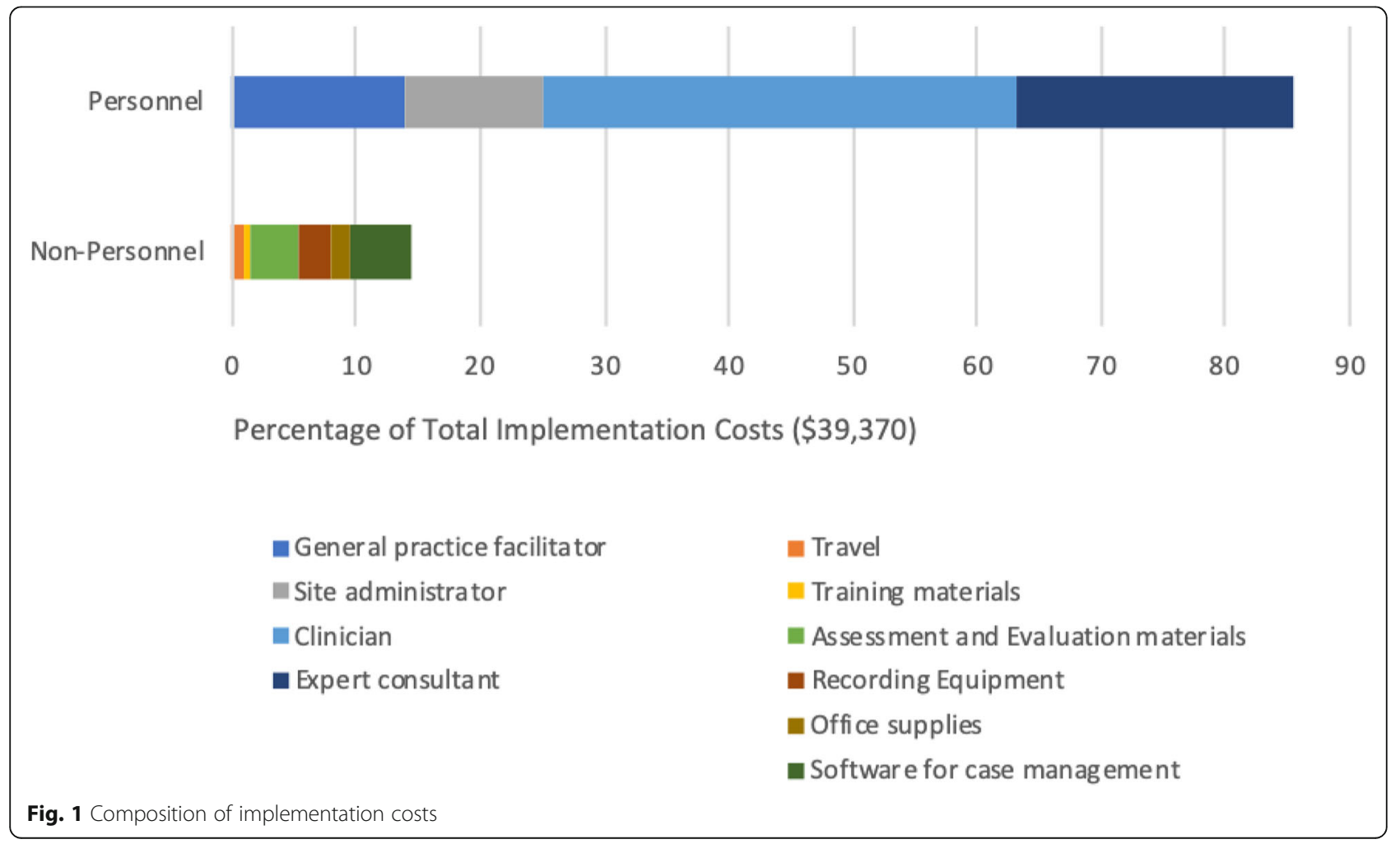




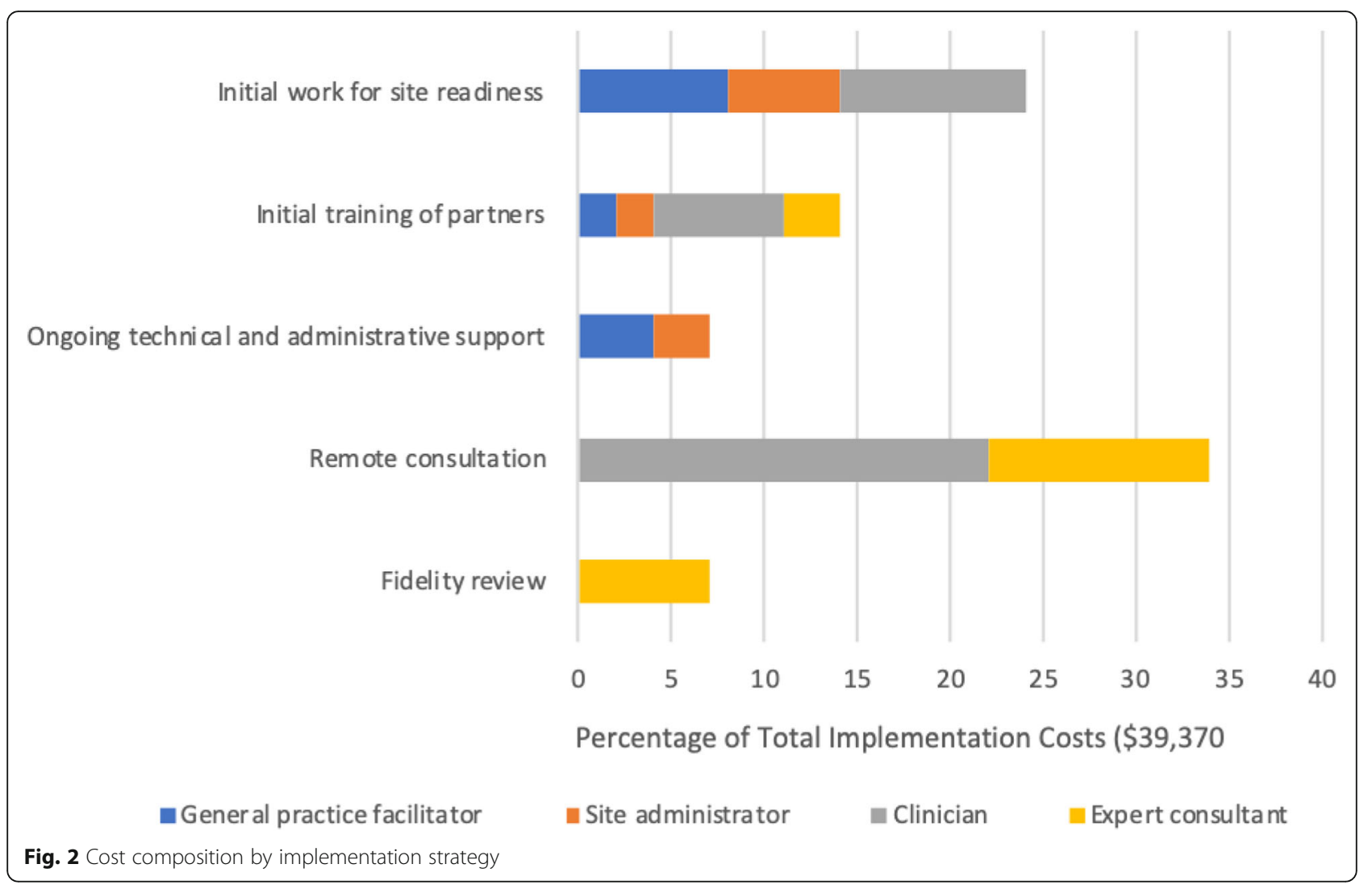

These visuals illustrate how the proposed method produces data that provides concrete and transparent cost information for decision-makers. It can support the identification of actions with little value, causes of variation in costs, and how changing a specific component would change costs. Detailed resource composition enables decisionmakers to look for the best balance among the resources allocated to the implementation strategies and to help identify places where it may be possible to reduce costs without substantially reducing effectiveness. For example, if it is apparent that travel time costs represent a large percentage of the overall cost burden, decision-makers may opt to use technology to conduct some meetings remotely rather than in person. Detailed information can also help to identify cases where it may be appropriate to shift specific tasks to less expensive personnel.

\section{Discussion}

In this study, we present a simple, pragmatic method for costing implementation strategies that combines time-driven activity-based costing, an established business accounting method, with the Proctor et al. framework, a well-known implementation science framework for specifying and reporting implementation strategies $[20,21$, 23]. Blending these two approaches allows for the integration of cost estimation into an existing structure that can guide evaluations of implementation strategy effectiveness. It involves clearly mapping implementation strategies by specifying the names, actions, actors, and temporality associated with them; determining the frequency and average duration of each action associated with individual strategies; and assigning a dollar value to the resources that each action consumes. Itemization of resource use and costs is piggy-backed on the Proctor framework; specifications of actions, actors, temporality, frequency, and average duration are used to directly determine the resources utilized by the strategies and their costs. Our approach allows researchers using the Proctor rubric to estimate implementation strategy costs and routinely report them alongside the other elements of the framework.

The detailed information produced by our approach benefits implementation strategy effectiveness research by providing a direct link between the implementation inputs (resources utilized) and implementation outcomes. Such information is 

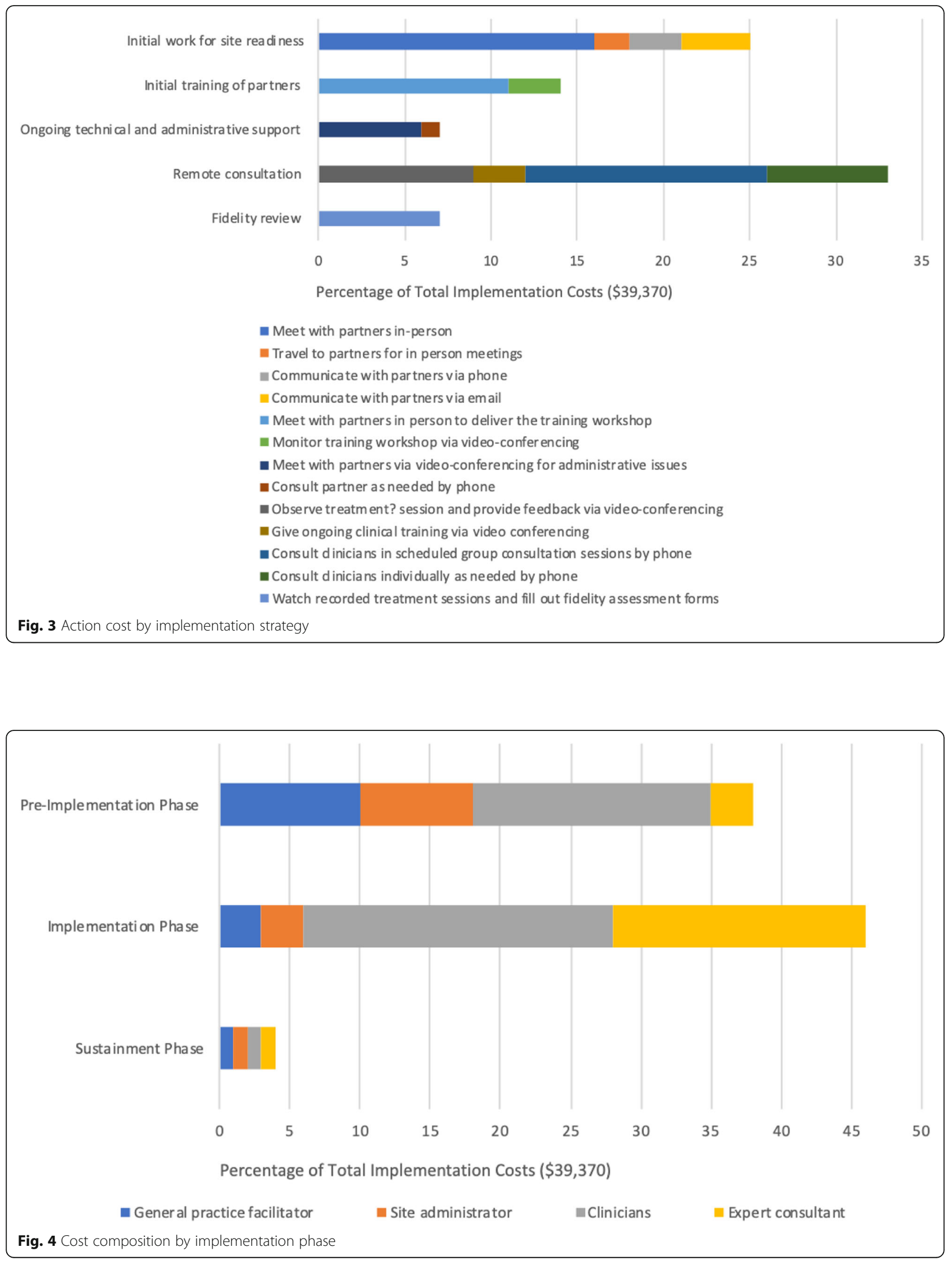
especially useful for studying the mechanisms and processes that determine the effects of an implementation strategy on an implementation outcome, which is another core element in the Proctor et al. framework. For example, the effect of the implementation strategy dosage, reflected by action frequency and unit duration of the actions, is an important area of inquiry in implementation science $[8,23,42]$. Another benefit of our approach is that it offers an important opportunity to study site differences in the actual implementation process, such as when there is variation in the delivery of implementation strategies across implementation sites. A TDABC resource matrix can be created for each site to reflect site-specific information, and crosssite comparisons can be conducted to identify variations in resource use and costs, such as may occur when a site needs more support. This element of the method is consistent with calls within implementation science to determine how strategy delivery varies across sites and to assess the implications of this variation for implementation outcomes.

Although several implementation science frameworks include costs as an important implementation outcome to be studied alongside other implementation measures, in practice, cost estimation has not been integrated into most evaluations of implementation strategy effectiveness $[5,8]$. The practicality of TDABC comes from its reliance on only two pieces of information that are often straightforward to determine: action frequency and average duration of unit action. In the context of implementation strategies, a majority of the relevant information can be obtained easily and accurately since the core activities that comprise an implementation strategy are typically specified in advance and then tracked and documented as part of implementation (e.g., documentation of consultation sessions for fidelity assessment) [38]. Thus, capturing this process data (e.g., number of consultation sessions, average duration of one consultation session) for costing purposes may not impose added burden. For standard, predictable or regular actions (e.g., weekly, 1-h stakeholder meetings; a 1-day training workshop), accurate data can be obtained easily if the actions are performed as planned. For more complex actions such as those of tailored implementation strategies, data can be obtained by direct observations as part of the process data. Applications of TDABC in organizational settings have demonstrated that determining activity frequencies and establishing an average duration is less burdensome than asking personnel to log their time and activities [15, 19, $21,27]$. The ability to observe these activities objectively likely makes documentation more accurate and reliable than personnel activity logs or general self-reported estimations of percentage effort. This is highly relevant in healthcare settings, where remembering to complete these logs may be especially challenging due to busy schedules and competing priorities.

As with all methods, this approach has limitations. Some activities, such as those requiring asneeded or ad hoc communication (e.g., phone calls, emails), are more difficult to capture in a standardized way and likely still require data collection via staff survey or logs, such as tracking communication frequency and average duration during a typical week during each implementation phase. Nonetheless, the majority of implementation activities are relatively straightforward to capture using this method (e.g., training workshops, scheduled consultation sessions, fidelity review sessions), thereby offering implementation science researchers an additional option for assessing costs via a systematic, detailed approach.

\section{Conclusion}

Availability of a step-by-step procedure to guide and standardize the application of TDABC in the costing of implementation strategies, following an implementation science framework that is widely used, may encourage academic researchers to perform TDABC costing studies and build our knowledge base in this area with detailed and transparent cost information and quality cost evaluation. This framework also provides an approach for how to better evaluate and control implementation procedures and associated costs, which should facilitate better decision-making when choosing implementation strategies and associated logistics.

Future research should test this method in costing implementation strategies, especially in cases where strategy actions can be granularized to represent multiple levels and components of the strategy. Efforts to build a TDABC toolkit to track implementation procedures and resource use would be useful, especially because many implementation strategies are likely to share common or routine actions. Just like prior efforts to compile implementation strategies with specific labels and taxonomies, efforts to compile a list of common actions across strategies would be useful to help researchers map the actions involved in implementation strategies. This would enhance efforts to standardize and systematize the study of implementation strategy costs. 


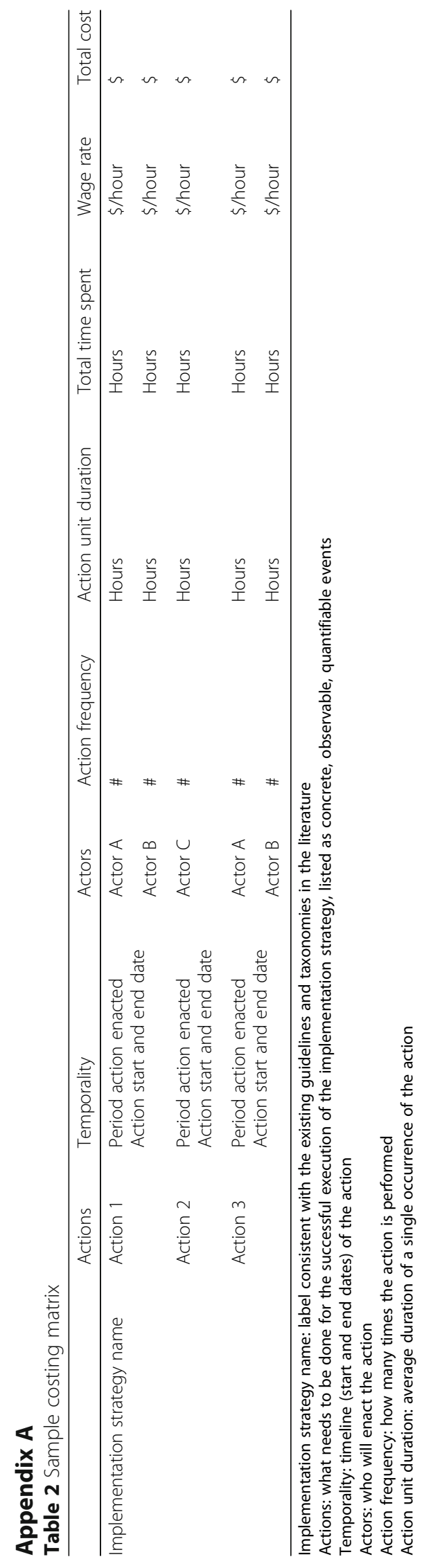




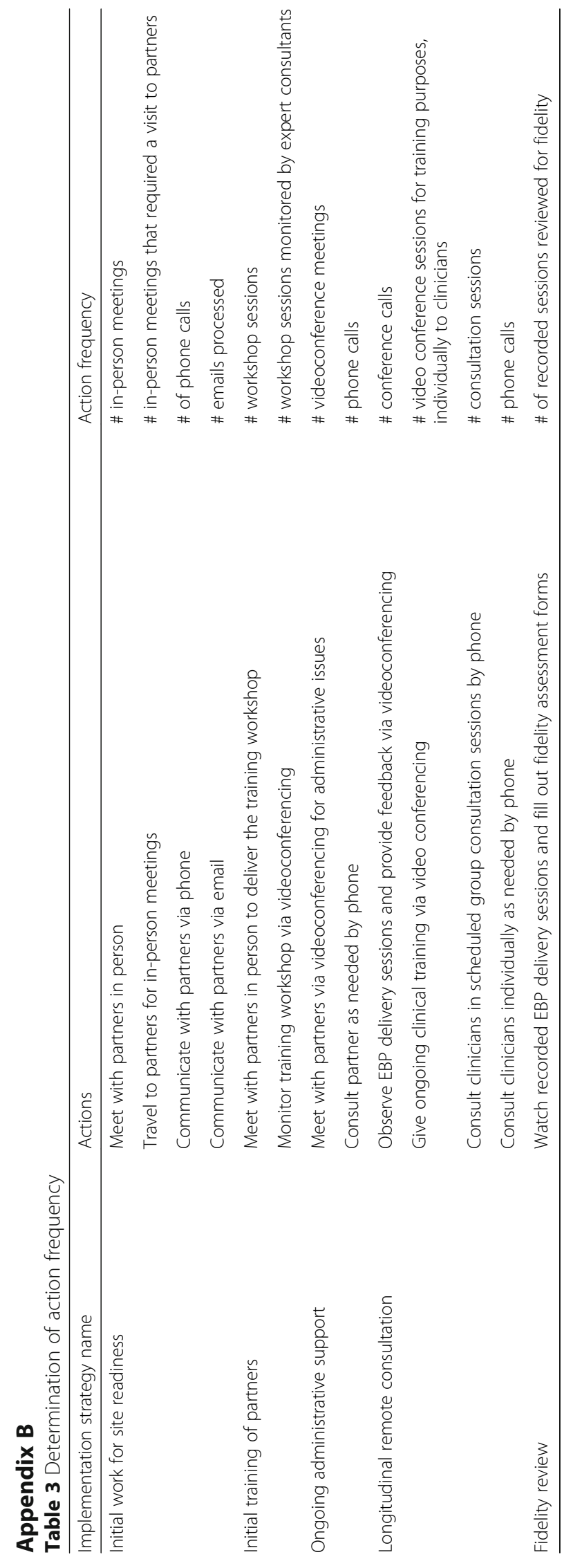




\section{Acknowledgements}

The authors thank Amy R. Pettit, PhD, for her feedback and assistance with the organization and editing of the manuscript.

\section{Authors' contributions}

ZC and SM conceptualized the study. DM provided important insights into the conceptual approach. ZC drafted the manuscript. All authors made important contributions to the intellectual context and interpreting insights. All authors read, edited, revised, and approved the final manuscript.

\section{Funding}

This work is funded by the National Institute of Mental Health (NIMH) (Project No: 3P50MH113840-03). Dr. Curran is supported by the Translational Research Institute (TRI), grants U54 TR001629 and UL1 TR003107, through the National Center for Advancing Translational Sciences of the National Institutes of Health $(\mathrm{NIH})$.

\section{Availability of data and materials}

\section{Not applicable.}

\section{Ethics approval and consent to participate} Not applicable.

\section{Consent for publication}

Not applicable.

\section{Competing interests}

The authors declare that they have no competing interests.

Dr. Beidas receives royalties from Oxford University Press and has provided consultation to Merck and the Camden Coalition of Healthcare Providers.

\section{Author details}

'Department of Psychiatry, Perelman School of Medicine, University of Pennsylvania, Philadelphia, PA, USA. ${ }^{2}$ Leonard Davis Institute of Health Economics, University of Pennsylvania, Philadelphia, PA, USA. ${ }^{3}$ Center for Mental Healthcare and Outcomes Research, Central Arkansas Veterans Healthcare System, North Little Rock, AR, USA. ${ }^{4}$ South Central Mental Illness Research, Education and Clinical Center, Central Arkansas, Little Rock, USA. ${ }^{5}$ Veterans Healthcare System, North Little Rock, AR, USA. ${ }^{6}$ Division of Health Services Research, Department of Psychiatry, College of Medicine, University of Arkansas for Medical Sciences, Little Rock, AR, USA. D. Department of Medical Ethics and Health Policy, Perelman School of Medicine, University of Pennsylvania, Philadelphia, PA, USA. ${ }^{8}$ Departments of Pharmacy Practice and Psychiatry, University of Arkansas for Medical Sciences, Little Rock, AR, USA. ${ }^{9}$ Center for Implementation Research, University of Arkansas for Medical Sciences, Little Rock, AR, USA. ${ }^{10}$ Central Arkansas Veterans Healthcare System, Little Rock, AR, USA. ${ }^{11}$ School of Social Policy and Practice, University of Pennsylvania, Philadelphia, PA, USA. ${ }^{2}$ Department of Medicine, Perelman School of Medicine, University of Pennsylvania, Philadelphia, PA, USA. ${ }^{13}$ Penn Implementation Science Center, Leonard Davis Institute of Health Economics, Philadelphia, USA.

\section{Received: 30 October 2019 Accepted: 16 April 2020} Published online: 05 May 2020

\section{References}

1. Aarons GA, Wells RS, Zagursky K, Fettes DL, Palinkas LA. Implementing evidence-based practice in community mental health agencies: a multiple stakeholder analysis. Am J Public Health. 2009;99:2087-95.

2. Bond GR, Drake RE, McHugo GJ, Peterson AE, Jones AM, Williams J. Longterm sustainability of evidence-based practices in community mental health agencies. Admin Pol Ment Health. 2014;41:228-36.

3. Willmeroth T, Wesselborg B, Kuske S. Implementation outcomes and indicators as a new challenge in health services research: a systematic scoping review. Inquiry. 2019;56:46958019861257.

4. Proctor E, Silmere H, Raghavan R, et al. Outcomes for implementation research: conceptual distinctions, measurement challenges, and research agenda. Admin Pol Ment Health. 2011;38:65-76.

5. Roberts SLE, Healey A, Sevdalis N. Use of health economic evaluation in the implementation and improvement science fields-a systematic literature review. Implement Sci. 2019;14:72.
6. Gold R, Bunce AE, Cohen DJ, et al. Reporting on the strategies needed to implement proven interventions: an example from a "real-world" crosssetting implementation study. Mayo Clin Proc. 2016;91:1074-83.

7. Dopp AR, Mundey P, Beasley LO, Silovsky JF, Eisenberg D. Mixed-method approaches to strengthen economic evaluations in implementation research. Implement Sci. 2019;14:2.

8. Powell BJ, Fernandez ME, Williams NJ, et al. Enhancing the impact of implementation strategies in healthcare: a research agenda. Front Public Health. 2019;7:3.

9. Eisman AB, Kilbourne AM, Dopp AR, Saldana L, Eisenberg D. Economic evaluation in implementation science: making the business case for implementation strategies. Psychiatry Res. 2020;283:112433.

10. O'Beirne M, Reid R, Zwicker K, Sterling P. The costs of developing, implementing, and operating a safety learning system in community practice. Journal of patient .... 2013.

11. Filene $\mathrm{JH}$, Brodowski ML, Bell J. Using cost analysis to examine variability in replications of an efficacious child neglect prevention program. J Publ Child Welfare. 2014;8:375-96.

12. Ritchie MJ, Kirchner JE, Townsend JC, Pitcock JA, Dollar KM, Liu CF. Time and organizational cost for facilitating implementation of primary care mental health integration. J Gen Intern Med. 2019.

13. Saldana L, Chamberlain P, Bradford WD, Campbell M, Landsverk J. The cost of implementing new strategies (COINS): a method for mapping implementation resources using the stages of implementation completion. Child Youth Serv Rev. 2014;39:177-82.

14. Liu CF, Rubenstein LV, Kirchner JE, et al. Organizational cost of quality improvement for depression care. Health Serv Res. 2009;44:225-44.

15. Keel G, Savage C, Rafiq M, Mazzocato P. Time-driven activity-based costing in health care: a systematic review of the literature. Health Policy. 2017;121:755-63.

16. Luce BR. Estimating costs in cost-effectiveness analysis. Cost-effectiveness in health and medicine. 1996.

17. Frick KD. Micro-costing quantity data collection methods. Med Care. 2009; 47:S76.

18. Charles JM, Edwards RT, Bywater T, Hutchings J. Micro-costing in public health economics: steps towards a standardized framework, using the incredible years toddler parenting program as a worked example. Prev Sci. 2013;14:377-89.

19. Areena SN. A review on time-driven activity-based costing system in various sectors. Journal of Modern Manufacturing Systems and .... 2019.

20. Kaplan RS, Anderson SR. Time-driven activity-based costing. Available at SSRN 485443. 2003.

21. Kaplan RS, Anderson SR. Time-driven activity-based costing: a simpler and more powerful path to higher profits; 2007.

22. Kaplan RS, Witkowski M, Abbott M, et al. Using time-driven activity-based costing to identify value improvement opportunities in healthcare. J Healthc Manag. 2014;59:399-412.

23. Proctor EK, Powell BJ, McMillen JC. Implementation strategies: recommendations for specifying and reporting. Implement Sci. 2013;8:139.

24. Busby JS, Williams GM. The value and limitations of using process models to describe the manufacturing organization. The International Journal of Production .... 1993.

25. Foster R, Blakely T, Wilson N, O'Dea D. Protocol for direct costing of health sector interventions for economic modelling (including event pathways). Wellington: University of Otago Department of Public Health; 2013.

26. da Silva Etges APB, Cruz LN, Notti RK, et al. An 8-step framework for implementing time-driven activity-based costing in healthcare studies. Eur J Health Econ. 2019;20:1133-45.

27. Gonzalez Ml. Time-driven activity-based costing for healthcare provider supply chain processes; 2014.

28. Huang Y-T. Evaluation and recommendation of implementing time-driven activity-based costing in healthcare [dissertation]. The University of Texas School of Public Health; 2016.

29. Powell BJ, McMillen JC, Proctor EK, et al. A compilation of strategies for implementing clinical innovations in health and mental health. Med Care Res Rev. 2012;69:123-57.

30. Powell BJ, Waltz TJ, Chinman MJ, et al. A refined compilation of implementation strategies: results from the Expert Recommendations for Implementing Change (ERIC) project. Implement Sci. 2015;10:21.

31. Mazza D, Bairstow $P$, Buchan $H$, et al. Refining a taxonomy for guideline implementation: results of an exercise in abstract classification. Implement Sci. 2013:8:32. 
32. Pinnock H, Barwick M, Carpenter CR, Eldridge S. Standards for reporting implementation studies (StaRI) statement. Bmj. 2017.

33. Boyd MR, Powell BJ, Endicott D, Lewis CC. A method for tracking implementation strategies: an exemplar implementing measurement-based care in community behavioral health clinics. Behav Ther. 2018:49:525-37.

34. Bunger AC, Powell BJ, Robertson HA, MacDowell H, Birken SA, Shea C. Tracking implementation strategies: a description of a practical approach and early findings. Health Res Policy Syst. 2017;15:15.

35. Grumbach K, Bainbridge E, Bodenheimer T. Facilitating improvement in primary care: the promise of practice coaching. Issue Brief (Commonw Fund). 2012;15:1-14

36. Kirchner JE, Smith JL, Powell BJ, Waltz TJ, Proctor EK. Getting a clinical innovation into practice: an introduction to implementation strategies. Psychiatry Res. 2020;283:112467.

37. Dopp AR, Parisi KE, Munson SA, Lyon AR. A glossary of user-centered design strategies for implementation experts. Transl Behav Med. 2019;9:1057-64.

38. Hulscher M, Wensing M. Process evaluation of implementation strategies. ... care: the implementation of change in .... 2020.

39. Aarons GA, Ehrhart MG, Farahnak LR, Hurlburt MS. Leadership and organizational change for implementation (LOCI): a randomized mixed method pilot study of a leadership and organization development intervention for evidence-based practice implementation. Implement Sci. 2015;10:11.

40. Aarons GA, Hurlburt M, Horwitz SM. Advancing a conceptual model of evidence-based practice implementation in public service sectors. Admin Pol Ment Health. 2011:38:4-23.

41. Giguère A, Légaré F, Grimshaw J, et al. Printed educational materials: effects on professional practice and healthcare outcomes. Cochrane Database Syst Rev. 2012;10:CD004398.

42. Huynh $A K$, Hamilton AB, Farmer MM, et al. A pragmatic approach to guide implementation evaluation research: strategy mapping for complex interventions. Front Public Health. 2018;6:134.

\section{Publisher's Note}

Springer Nature remains neutral with regard to jurisdictional claims in published maps and institutional affiliations.

Ready to submit your research? Choose BMC and benefit from:

- fast, convenient online submission

- thorough peer review by experienced researchers in your field

- rapid publication on acceptance

- support for research data, including large and complex data types

- gold Open Access which fosters wider collaboration and increased citations

- maximum visibility for your research: over $100 \mathrm{M}$ website views per year

At $\mathrm{BMC}$, research is always in progress.

Learn more biomedcentral.com/submissions 